\title{
Heart Rate Variability and Mild Cognitive Impairment in Parkinson's Disease
}

Cinthia Terroba-Chambi, MD,1,2

Carolina Abulafia, $\mathrm{PhD}, 1,3$

Daniel E. Vigo, MD, PhD, 3,4

Marcelo Merello, MD, PhD1,3 mmerello@fleni.org.ar

1 Raul Carrea Institute of Neurological Research, Movement

Disorders Unit, FLENI, Buenos Aires, Argentina,

2 National Scientific

and Technological Research Council (CONICET), Buenos Aires, Argentina,

3 Institute for Biomedical Research, Pontifical Catholic

University of Argentina (UCA), Buenos Aires, Argentina, and

4 Faculty of Psychology and Educational Sciences, Katholieke Universiteit Leuven, Leuven, Belgium

Heart rate variability (HRV) is an index of autonomic control of the heart.1 Evidence suggests loss of sympathetic innervation of the heart takes place in early stages of Parkinson's disease (PD) and is independent of orthostatic hypotension.2,3 Information about the relationship between HRV and mild cognitive impairment in PD (PD-MCI) is scarce. 4 We assessed the association between short-term HRV and MCI in participants of a larger observational crosssectional study of fear of falling. The local ethics committee approved the study, and patients provided written informed consent. Patients without dementia with idiopathic PD and stable medication regimen were included, excluding those with cardiac, liver, or kidney diseases and medications (eg, betareceptor blockers) that affect HRV. Patients were examined during ON state. Levodopa equivalent dose (LED) and blood pressure measures were calculated. Subjects were classified as possible 
PD-MCI by Movement Disorder Society criteria.5 Electrocardiogram signal was recorded on subjects resting for 10 minutes, and a second for 5 minutes under the same conditions [resting state (RS)] and for an additional 5 minutes [standing state (SS)]. Time and frequency domains analysis involved mean duration of RR intervals, standard deviation of all RR normal intervals, square root of the mean of the sum of the squares of differences between adjacent RR intervals (RMSSD), power in the low-frequency range, power in the high-frequency range, normalized high-frequency range, and lowfrequency/high-frequency range ratio. Nonlinear parameters included short-term detrended fluctuation analysis (DFA $\alpha 1)$ and sample entropy.6 Statistical analysis was performed using IBM SPSS Statistics v23.0 ( IBM Corp., NY, USA). Medians and interquartile ranges were calculated for each group. Unpaired Mann-Whitney U tests were used for continuous variables, and $\chi^{2}$ tests were used for categorical variables. Correlations were calculated with Spearman's rho. Benjamini-Hochberg's false discovery rate correction was applied to multiple intergroup comparisons.

There were no significant differences in demographic, clinical, or blood pressure variables (Table 1). Patients with MCI showed lower RMSSD than the non-MCI group in RS [18.7 (12.1-25.0) versus 32 (20.6-57.7); $\mathrm{P}=0.047]$. No significant differences were observed during SS. The MCI group exhibited a statistically significant reduction in RMSSD in SS-RS differences, although it did not survive false discovery rate correction.

Our findings show reduced RMSSD in patients with MCI, indicative of decreased parasympathetic activity. The absence of significant differences in other HRV measures could be explained by subtle autonomic changes or reduced statistical power. The role of HRV in cognition showed a reciprocal relationship between autonomic nervous system and central cognitive function based on the Neurovisceral Integration Model7 linking 
HRV, prefrontal neural structures, and frontal and midbrain areas, which reflect autonomic nervous system brain distribution and its relationship with cognition. The generalizability of our findings may be limited because of our small sample size, crosssectional design, the confounding effects of levodopa, and the lack of an extensive cognitive examination.

This pilot study is the first to describe the association between short-term HRV and cognition in patients without dementia with PD, suggesting lower parasympathetic activity is associated with MCI independently of blood pressure changes during the orthostatic challenge, which supports HRV as a possible independent autonomic marker of PDMCI. Its role as a predictive biomarker of PD-MCI should be explored in larger studies.

Table 1. Clinical and demographic information

\begin{tabular}{|c|c|c|c|c|}
\hline & Total $(n=20)$ & Non- $\mathrm{MCl}(\mathrm{n}=7)$ & $\mathrm{MCl}(n=13)$ & $P$ \\
\hline $\mathrm{Age}^{\mathrm{a}}(\mathrm{yr})$ & $72.5(64-75)$ & $72(65-75)$ & $73(63-75)$ & 0.817 \\
\hline Gender (male:female) & $11: 9$ & $5: 2$ & $6: 7$ & 0.279 \\
\hline Illness duration ${ }^{\mathrm{a}}$ (yr) & $6.5(4-11)$ & $9(4-14)$ & $6(4-10)$ & 0.643 \\
\hline Formal education $\mathrm{n}^{\mathrm{a}}(\mathrm{yr})$ & $13(12-17)$ & $17(12-17)$ & $12(12-17)$ & 0.393 \\
\hline $\mathrm{BMI}^{\mathrm{a}}$ & $26.9(24.6-29.6)$ & $28.6(26.3-33.2)$ & $26.3(24.3-27.9)$ & 0.081 \\
\hline $\mathrm{LED}^{\mathrm{a}}(\mathrm{mg})$ & $750(570-1,117.5)$ & $580(400-925)$ & $1,000(712.5-1,250)$ & 0.057 \\
\hline MDS-UPDRS $1^{\mathrm{a}}$ & $7.5(4-12.5)$ & $5(3-13)$ & $9(7-12)$ & 0.351 \\
\hline MDS-UPDRS $\|^{\mathrm{a}}$ & $9(5-16.5)$ & $6(3-22)$ & $11(6-16)$ & 0.643 \\
\hline MDS-UPDRS $\|^{\mathrm{a}}$ & $31.5(26.5-34)$ & $29(25-33)$ & $32(28-35)$ & 0.757 \\
\hline MDS-UPDRS IV ${ }^{a}$ & $0(0-1.5)$ & $0(0-1)$ & $0(0-2)$ & 0.485 \\
\hline Hoehn \& Yahr ${ }^{a}$ & $2(2$ to 2$)$ & 2 (2 to 2$)$ & 2 (2 to 2$)$ & 0.817 \\
\hline Barthel Index ${ }^{a}$ & $85(78-95)$ & $90(75-95)$ & $85(80-95)$ & 0.938 \\
\hline $\mathrm{MoCA}^{\mathrm{a}}$ & $24(23-27.5)$ & $28(27-29)$ & $24(20-24)$ & $<0.001^{*}$ \\
\hline $\mathrm{FAB}^{\mathrm{a}}$ & $15.5(12.5-17)$ & $17(16-18)$ & $15(11-16)$ & $0.008^{\star}$ \\
\hline TMT-Ba $(\mathrm{s})$ & 126.5 (109.5-190.5) & $118(88-196)$ & $129(117-185)$ & 0.275 \\
\hline SDMT $^{\mathrm{a}}$ & $25(22-31)$ & $26(25-35)$ & $23(22-30)$ & 0.211 \\
\hline PVF $^{\mathrm{a}}$ & $13.5(11-16)$ & $16(14-19)$ & $11(9-15)$ & $0.024^{\star}$ \\
\hline Stroop $p^{a}$ & $-3.9(-5.9$ to 1.8$)$ & $2.2(-4.5$ to 9.1$)$ & $-4.7(-7.1$ to -1.3$)$ & $0.019^{*}$ \\
\hline $\mathrm{BDI} \|^{\mathrm{a}}$ & $15(10-18)$ & $15(7-16)$ & $16(11-19)$ & 0.275 \\
\hline$\left.B A\right|^{\mathrm{a}}$ & $12(5-15)$ & $11(2-13)$ & $12(6-16)$ & 0.536 \\
\hline $\mathrm{SAS}^{\mathrm{a}}$ & $11(6-19)$ & $8(1-21)$ & $13(8-16)$ & 0.351 \\
\hline \multicolumn{5}{|l|}{ BPM } \\
\hline $\mathrm{OH}^{\mathrm{b}}$ & $8(40)$ & $2(29)$ & $6(46)$ & 0.260 \\
\hline $\mathrm{NOH}^{\mathrm{b}}$ & $6(30)$ & $1(14)$ & $5(39)$ & 0.444 \\
\hline $\mathrm{SBP}^{\mathrm{a}}$ supine $(\mathrm{mm} \mathrm{Hg})$ & $120(110-127.5)$ & $120(110-120)$ & $120(110-130)$ & 0.689 \\
\hline $\mathrm{SBP}^{\mathrm{a}}$ standing $(\mathrm{mm} \mathrm{Hg})$ & $120(115-130)$ & $120(110-120)$ & $120(120-130)$ & 0.485 \\
\hline $\mathrm{DBP}^{\mathrm{a}}$ supine $(\mathrm{mm} \mathrm{Hg})$ & $77.5(70-80)$ & $70(60-80)$ & $80(70-80)$ & 0.211 \\
\hline $\mathrm{DBP}^{\mathrm{a}}$ standing (mm Hg) & $80(70-85)$ & $80(70-80)$ & $80(70-85)$ & 0.701 \\
\hline MAP $^{\mathrm{a}}$ supine $(\mathrm{mm} \mathrm{Hg})$ & $91.7(81.7-95.3)$ & 90 (76.7-93.3) & $93.3(83.3-98.3)$ & 0.311 \\
\hline $\mathrm{MAP}^{\mathrm{a}}$ standing $(\mathrm{mm} \mathrm{Hg})$ & $93.3(85-100)$ & $93.3(86.7-100)$ & $96.7(83.3-100)$ & 0.699 \\
\hline
\end{tabular}

*Significant at $\mathrm{P}<0.05$ comparisons Mann-Whitney $\mathrm{U}$ test or $\chi 2$ test (degrees of freedom $=1.20$ ). aMedian (interquartile range, $25 \%-75 \%$ ).

bn (\% of column). Comparisons surviving false discovery rate correction are indicated in bold. 
Abbreviations: $\mathrm{MCl}$, mild cognitive impairment; BMI, body mass index; LED, levodopa equivalent dose; MDS-UPDRS, Movement Disorder Society Unified Parkinson's Disease Rating Scale; MDS-UPDRS I, MDSUPDRS part I, nonmotor symptoms subscore; MDS-UPDRS II, MDS-UPDRS part II, motor symptoms subscore; MDS-UPDRS III, MDS-UPDRS part III, motor subscore; MDS-UPDRS IV, MDS-UPDRS part IV, complications subscore; MoCA, Montreal Cognitive Assessment; FAB, Frontal Assessment Battery; TMT$B$, Trail Making Test part B; SDMT, symbol digit modality test; PVF, phonemic verbal fluency letter " $p$ "; BDI II, Beck Depression Inventory, second edition; BAI, Beck Anxiety Inventory; SAS, Starkstein Apathy Scale; BPM, blood pressure measures; $\mathrm{OH}$, orthostatic hypotension; $\mathrm{NOH}$, neurogenic orthostatic hypotension ( $\triangle \mathrm{HR} / \triangle \mathrm{BP} \leq 0.49, \mathrm{HR}$, heart rate; $\mathrm{BP}$, blood pressure); $\mathrm{SBP}$, systolic blood pressure; $D B P$, diastolic blood pressure; MAP, mean arterial pressure $(\mathrm{MAP}=\mathrm{SBP}+2(\mathrm{DBP}) / 3)$.

\section{References}

1. Forte G, Favieri F, Casagrande M. Heart rate variability and cognitive function: a systematic review. Front Neurosci 2019;13:710.

2. Barbic F, Perego F, Canesi M, et al. Early abnormalities of vascular and cardiac autonomic control in Parkinson's disease without orthostatic hypotension. Hypertension 2007;49(1):120-126.

3. Haensch CA, Lerch H, Jörg J, Isenmann S. Cardiac denervation occurs independent of orthostatic hypotension and impaired heart rate variability in Parkinson's disease. Parkinsonism Relat Disord 2009;15(2):134-137.

4. Barbieri R, Citi L, Guerrisi M, et al. Lower instantaneous entropy of heartbeat dynamics characterizes cognitive impairment in Parkinson's disease. Computing in Cardiology Conference (CinC), IEEE. 2014: $81-84$.

5. Litvan I, Goldman JG, Tröster AI, et al. Diagnostic criteria for mild cognitive impairment in Parkinson's disease: Movement Disorder Society Task Force guidelines. Mov Disord 2012;27(3):349-356.

6. Terroba-Chambi C, Bruno V, Vigo DE, Merello M. Heart rate variability and falls in Huntington's disease. [published online ahead of print, 2020 Feb 6]. Clin Auton Res 2020. https://doi.org/10.1007/

s10286-020-00669-2.

7. Thayer JF, Lane R. A model of neurovisceral integration in emotion regulation and dysregulation. J Affect Disord 2000;61(3):201-216. 